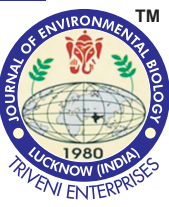

\title{
Selection of reference genes for RT-qPCR analysis in the bark of Populus yunnanensis cuttings
}

\begin{tabular}{|c|c|}
\hline Paper received: 17. 04. 2018 & Revised received: 14.11 .2018 \\
\hline
\end{tabular}

\section{Authors Info \\ T. Yun ${ }^{1,2}$, J. Li ${ }^{1,2}, Y . X u^{1,2}$, A. Zhou ${ }^{1,2}$, D. Zong ${ }^{1,2}$, S. Wang ${ }^{1,2}$, D. $\mathrm{Li}^{3}$ and C. $\mathrm{He}^{1,2,4 *}$ \\ ${ }^{1}$ Key Laboratory for Forest Genetic and Tree Improvement and \\ Propagation in Universities of Yunnan Province, Southwest \\ Forestry University, \\ Kunming-650 224, China \\ ${ }^{2}$ Key Laboratory of Biodiversity Conservation in Southwest China, State Forestry Administration, \\ Southwest Forestry University, Kunming-650 224, China \\ 3Yunnan Academy of Biodiversity, Southwest Forestry University, Kunming-650 224, China \\ ${ }^{4}$ Key Laboratory for Forest Resources Conservation and Use in the Southwest Mountains of China, Ministry of Education, Southwest Forestry University, Kunming-650224, China \\ *Corresponding Author Email : hcz70@163.com}

\section{Edited by}

Prof. Muhammad Aqeel Ashraf

Reviewed by

Dr. Luo Qiang

Dr. Wan Zhen

\section{Abstract}

Aim: The aim of this paper was to select and evaluate the stability of seven candidate reference genes (PDE1, TUA2, UBQ, ACT2, TUB, HIS and 18SRNA) under three different conditions for RT-qPCR analysis.

Methodology: Cuttings of Populus yunnanensis were cultured in water by two ways, upright and inverted vertically. Different internode barks were collected as test materials from 0 day to 49 day, once in seven day. Upright samples $(U)$ and inverted samples $(I)$ are two different conditions, total samples $(U+I)$ means the third condition. Seven candidate reference genes (PD-E1, TUA2, UBQ, ACT2, TUB, HIS and 18S RNA) were amplified by RT-qPCR. geNorm, NormFinder, BestKeeper and RankAggreg programs were used to select and evaluate the stability of seven candidate reference genes under three different conditions.

Results: The final combination of multiple reference genes to normalize gene expression under different conditions were HIS + $P D-E 1+T U B$ in upright samples (U), $P D-E 1+T U A 2$ in inverted samples (I), $P D-E 1+H I S+T U A 2$ in total samples $(U+I)$.

Interpretation: Stable reference genes could ensure RT-qPCR results correct by. The results provide an important reference gene selection guide in barks of P.yunnanensis cuttings for genes expression research.

Key words: Populus y unn ane nsis, Reference gene, RTqPCR, Intermode barks

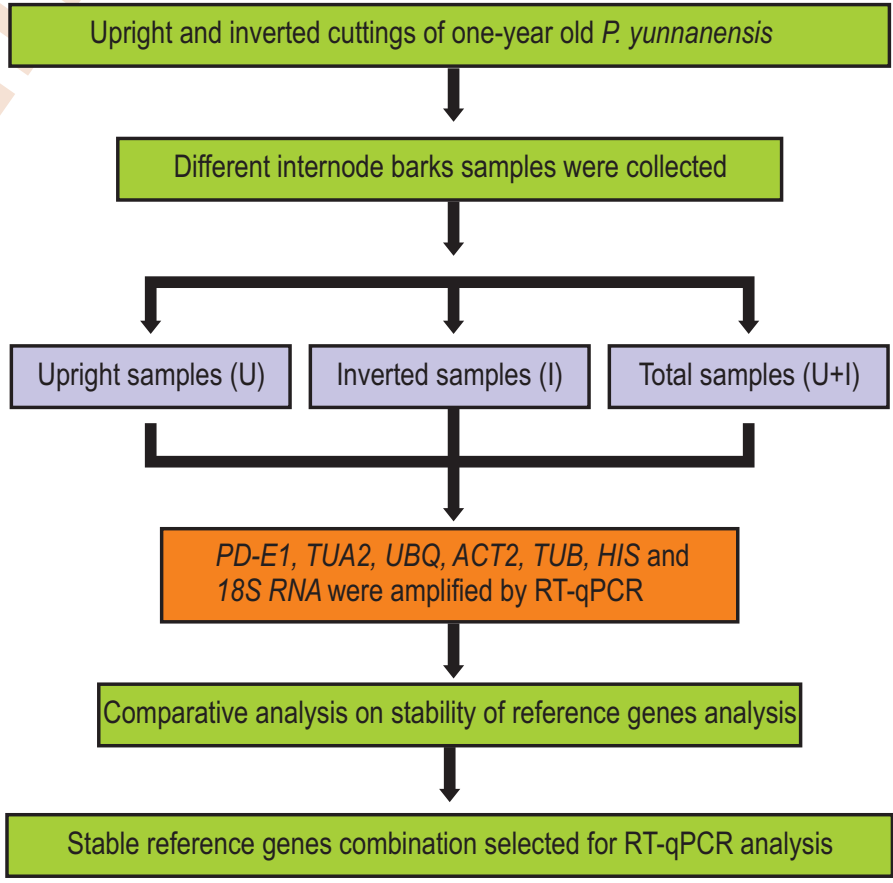

Citation: Yun, T., J. Li, Y. Xu, A. Zhou, D. Zong, S. Wang, D. Li and C. He: Selection of reference genes for RT-qPCR analysis in the bark of Populus yunnanensis cuttings, 40, 584-591 (2019). 


\section{Introduction}

Populus yunnanensis with fast growing, easy cultivation and extensive adaptability characteristic, is a unique Tacamahaca section poplar species in Southwest China, which has been widely used in afforestation. Due to lack of female samples in natural habitats, existing $P$. yunnanensis plantations are mainly formed by cuttings of male branches (He et al., 2004). Limited by auxin polar transport, plant cuttings should be consistent with the morphological direction and should avoid inversion (Grebe, 2004; Sachs, 1991; Sultana et al., 2018). However, our previous studies have found that the adventitious roots formed at the original morphological apex and shoots sprouted at the original morphological base of inverted cuttings, and inverted cuttings normally develop into intact plants (Li et al., 2015; Kibria et al., 2018). In further study, expression of different genes in the bark of P.yunnanensis between upright and inverted cuttings will be analyzed by reverse transcription-quantitative polymerase chain reaction (RT-qPCR).

RT-qPCR is one of the most widely used methods for gene expression studies in mRNA levels, because of it's sensitivity, specificity, accuracy and cost-effectiveness (Gachon et al., 2004; Nolan et al., 2006; Patil et al., 2017). However, gene expression data obtained using RT-qPCR can be affected by different variables, such as quality and amount of starting material, RNA quality (purity, integrity, DNA contamination), reverse transcription efficiency and amplification efficiency etc. (Pfaffl et al., 2004; Vandesompele et al., 2002; Abbas et al., 2018). Therefore, reference genes (internal control genes) are simultaneously applied to compensate these variations (Kumar et al., 2018; Park et al., 2015; Suzuki et al., 2000). Housekeeping genes such as $18 \mathrm{~S}$ ribosomal RNA (18S rRNA), actin (ACT), tubulin (TUB), ubiquitin (UBQ), histone (HIS) and glyceraldehyde-3-phosphate dehydrogenase (GAPDH) are often used as traditional reference genes to normalize mRNA expression because of their constitutive expression or their involvement in maintenance of cell structure(Castonguay et al., 2015; Grbić etal., 2011; Khan et al., 2018; Li etal., 2018).

However, these genes have variable expression patterns across tissue types and experimental conditions. Therefore, selection of suitable reference genes is important to avoid incorrect results obtained from differential expression patterns in specific tissue types and experimental conditions (Dheda et al., 2005; Solanas et al., 2001).In addition, using a single reference gene may go against obtaining subtle changes in genes expression, hence, many researchers tend to use geometric mean of multiple reference genes to ensure correct results (Carraro et al., 2012; Gutierrez et al., 2008; Schmid et al., 2003; Lu et al., 2018). Therefore, under certain experimental materials and conditions, choose stable reference gene and how many reference genes should be used in RT-qPCR experiments has become a key issue for consideration.
This study was carried out with the aim to select and evaluate the stability of seven candidate reference genes, using bark samples from different region of the upright and inverted cuttings. The stability of $P D-E 1$ (Pyruvate Dehydrogenase E1), TUA2 ( $\alpha$-tubulin), UBQ (poly ubiquitin enzyme), ACT2 (actin), TUB ( $\beta$-tubulin), HIS (histone), 18S RNA (18S ribosomal RNA) were analyzed byge Norm (Vandesompele et al., 2002), Norm Finder (Andersen et al., 2004),BestKeeper (Pfaffl et al., 2004)and RankAgregg (Pihur et al., 2009) programs, respectively. The final combination of reference genes used under different experiment conditions were obtained by the above comprehensive results.

\section{Materials and Methods}

Plant materials and treatments: All cuttings of one-year old $P$. yunnanensis $60 \sim 70 \mathrm{~cm}$ of length were collected from Haikou forest farm, Kunming, China and taken to laboratory for upright and inverted water culture without other nutrition in tub, upright water culture to keep cuttings vertical as morphological direction, conversely, inverted water culture to keep cuttings vertical in the opposite of morphological direction, replace the water once two days. Duration of water culture, nine plump lateral buds were retained above water for leaf morphological development. Upper,

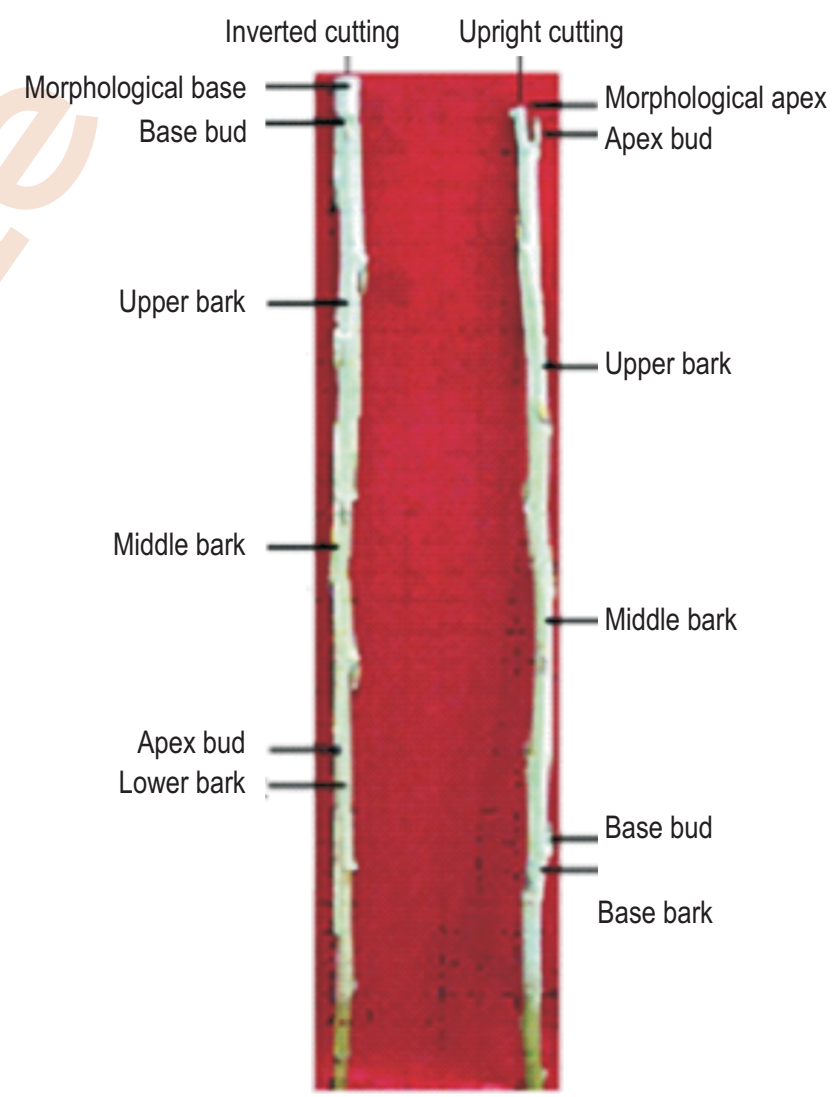

Fig. 1 : Upper, middle and lower bark was located in the position between the $3^{\text {rd }}$ to $4^{\text {th }}$ lateral bud, $6^{\text {th }} \sim 7^{\text {th }}$ lateral bud and $1 \mathrm{~cm}$ lower $9^{\text {th }}$ lateral bud, respectively. The collected bark samples include the cortex, phloem and cambium tissues. 
Table 1: Primers of the candidate reference genes used for RT-qPCR

\begin{tabular}{|c|c|c|c|c|}
\hline Gene & Forward primer(5' to $\left.3^{\prime}\right)$ & Reverse primer( $5^{\prime}$ to $\left.3^{\prime}\right)$ & Product size/bp & Reference \\
\hline$P D-E 1$ & ATGAGAACTGGTGGTATTGGTGC & GTCACAATCTGGGCAGGTTGAAC & 164 & (Carraro et al., 2012) \\
\hline TUA2 & СCTACTGTAGTACCTGGGGGTG & ССААСТTССТСGТААТССТTСТСА & 230 & (Carraro et al., 2012) \\
\hline UBQ & CAGCTTGAAGATGGGAGGAC & CAATGGTGTCTGAGCTCTCG & 154 & (Secchi et al., 2009) \\
\hline ACT2 & GCAACTGGGATGATATGGAGA & TACGACCACTGGCATACAGG & 213 & (Secchi etal., 2009) \\
\hline TUB & GCACCAACTTGTTGAGAATGC & TTTCAACTGACCAGGGAACC & 175 & (Brunner etal., 2004) \\
\hline HIS & TTTAAGACTGATCTGCGTTTCC & GAACAGCCCAACAAGGTATG & 95 & (Shuai et al., 2014) \\
\hline $18 S r R N A$ & TCAACTTTCGATGGTAGGATAGTG & CCGTGTCAGGATTGGGTAATTT & 145 & (Shuai et al., 2014) \\
\hline
\end{tabular}

middle and lower barks (Fig. 1) were collected after culture 0 day, 7 day, 14 day, 21 day, 28 day, 35 day, 42 day and 49 day, respectively, until the cuttings withered. Mix three cuttings equal proportions barks of the same position as a biological replicates, total of three mixed biological replicates. Upright samples $(U)$ and inverted samples (I) are two different conditions, total samples ( $U$ + I) means third condition. All samples were frozen in liquid nitrogen immediately after harvest and stored at $-80^{\circ} \mathrm{C}$ until future use.

Total RNA isolation and cDNA preparation: Total RNA was subsequently extracted using the E.Z.N.A. ¿ ${ }^{\circledR}$ Plant RNA Kit (OMEGA, USA), according to manufacturer's manual. Genomic DNA was eliminated by RNase-Free DNase I (OMEGA, USA) according to manual. The RNA concentration and purity were evaluated with Thermo Nano Drop 2000 (Thermo, USA). Integrity of RNA was verified by $1.2 \%$ (w/v) agarose gels. Only RNA samples with A260/A280 ratios between 1.8 and 2.2 and A260/A230 ratios higher than 2.0, as well as both $28 \mathrm{~S}$ and $18 \mathrm{~S}$ ribosomal RNA bands with a density ratio about 2:1 were used for further analysis. The first strand cDNA was synthesized from equal amounts of purified total RNA $(1.5 \mu \mathrm{g})$, using the Quantscript RT Kit (TIANGEN, China) as prescription manufacturer's manual with oligo-dT15 primer. All cDNA samples were stored at $-20^{\circ} \mathrm{C}$ and diluted $1: 8(\mathrm{v} / \mathrm{v})$ with RNase-free water before being used as template in RT-qPCR analysis.

Primer obtain and RT-qPCR amplification: RT-qPCR primer pairs were listed in Table 1, which were commonly used as internal control for gene expression studies in Populus. All the primer pairs were custom ordered from a commercial supplier (Sangon, Shanghai). PCR products of desired size were analyzed on $2 \%$ agarose gel, and then sequenced date amplification specificity was checked by BLAST search in NCBI. RT-qPCR was performed using Roche LightCycler®96 System (Roche, Swiss) and the SYBR® FAST qPCR Kit (KAPA, USA). The PCR reaction volume was $20 \mu$ containing 10 $\mu$ l 2x KAPA SYBR@ FAST qPCR Master Mix, $0.8 \mu$ l of each $5 \mu \mathrm{M}$ primer, $1 \mu \mathrm{l}$ CDNA and $7.4 \mu \mathrm{ddH}_{2} \mathrm{O}$. The RT-qPCR amplification cycle conditions of seven candidate reference genes were set as follows: $95^{\circ} \mathrm{C}$ for $3 \mathrm{~min}$, followed by 40 cycles of $95^{\circ} \mathrm{C}$ for $10 \mathrm{sec}$, $57^{\circ} \mathrm{C}$ for $20 \mathrm{sec}$. This cycle was followed by a melting curve analysis as $95^{\circ} \mathrm{C}$ for $10 \mathrm{sec}, 65^{\circ} \mathrm{C}$ for $60 \mathrm{sec}, 97^{\circ} \mathrm{C}$ for $1 \mathrm{sec}$.

Data analysis: Expression levels of the tested reference genes were determined by Ct values obtained by Light Cycler®96 1.1 software. Each average $\mathrm{Ct}$ value of three mixed biological replicates was calculated in Excel as data analysis base. GeNorm, Norm Finder and Best Keeper programs were used to analyze the express stability of candidate reference genes in upright samples (U), inverted samples (I), and total samples (U + I), respectively,in order to explore the effects of different treatment conditions on the stability of the candidate reference genes. RankAggreg was used to obtain the final combination of multiple reference genes under three different conditions. Best Keeper software can directly analyze the raw average $\mathrm{Ct}$ values, which should been converted to scale expression quantities for geNorm and Norm Finder analysis. As the highest relative quantity (the minimum Ct value) for each gene was set to 1 , the other average $\mathrm{Ct}$ values of each duplicate reaction of sample was converted to 2- $\Delta \mathrm{Ct}(\Delta \mathrm{Ct}=$ sample $\mathrm{Ct}-\min \mathrm{Ct})$.

\section{Results and Discussion}

GeNorm analysis: GeNorm program, a Visual Basic application tool for Microsoft Excel, measures gene expression stability (M value), which is the mean pair-wise variation between an individual gene and all other tested control genes. GeNorm program ranks the genes based on their M values; the genes with high $M$ values are less stably expressed and make a bad reference gene, whereas those with low $M$ values are stably expressed and make a good reference gene (Vandesompele et al., 2002). The range of expression stability calculated by geNorm in the genes analysed was as follows (from the most stable to the least stable): PD-E1/TUA2, HIS, TUB, UBQ, ACT2, 18S rRNA in upright samples $(U)$, as the same within inverted samples $(I)$ and in total samples $(U+I)$ (Fig.2).

In addition, the geNorm program can calculate the optimal number of required reference genes for obtaining reliable results from RT-qPCR studies. This calculation was performed by analysis of pair-wise variation $(V n / n+1)$ of sequential normalization factors (NF) with an increasing number of reference genes (NFn and NFn+1). The geNorm program proposed 0.15 as a cut-off value, below which the inclusion of an 
Average expression stability values of remaining control genes

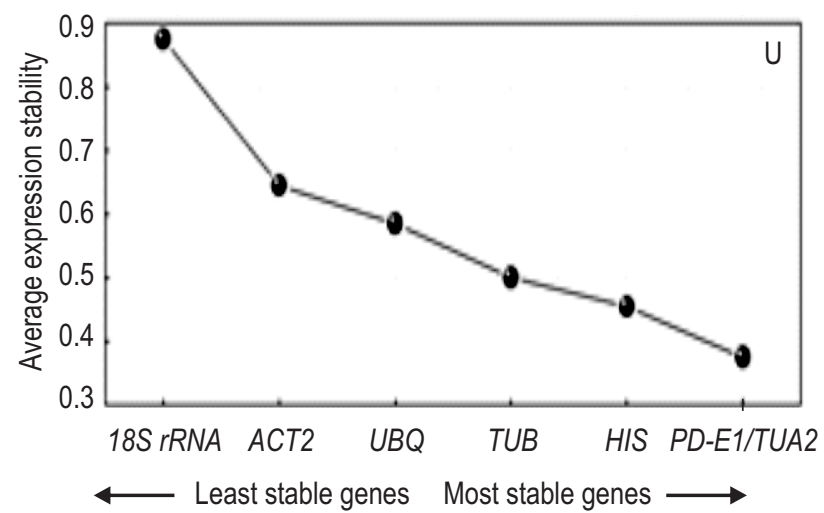

Average expression stability values of remaining control genes

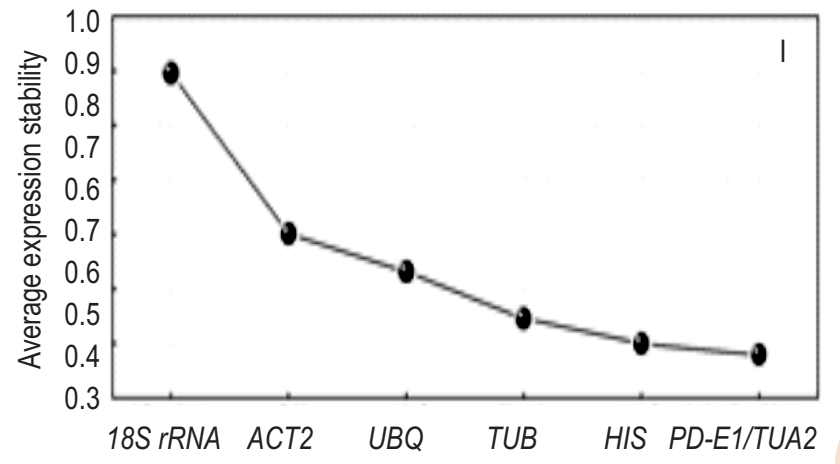

$\longleftarrow$ Least stable genes Most stable genes $\longrightarrow$

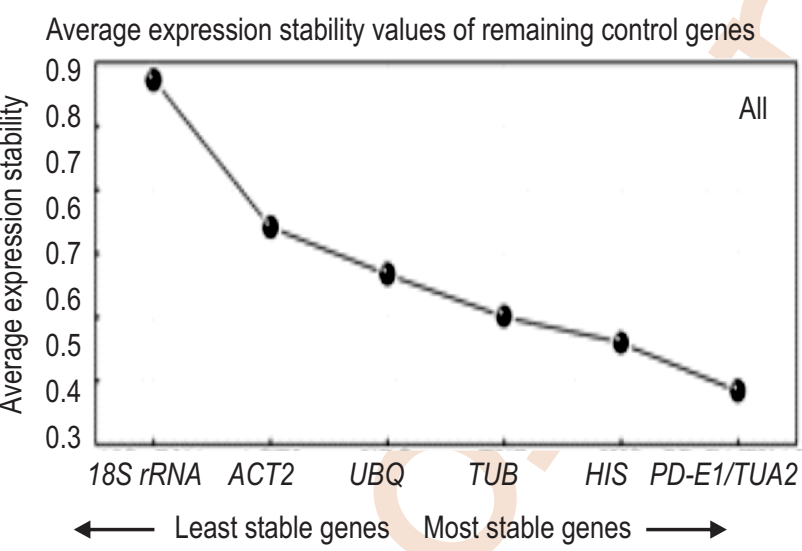

Determination of the optimal number of control genes for normalization

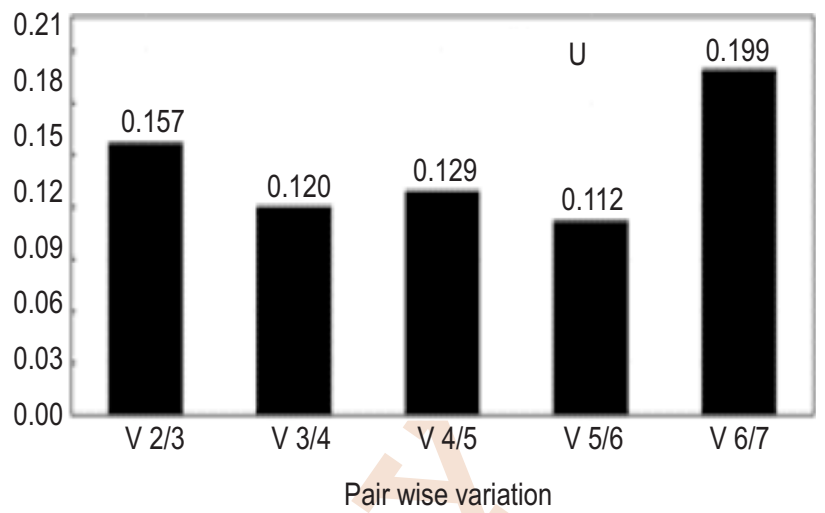

Determination of the optimal number of control genes for normalization

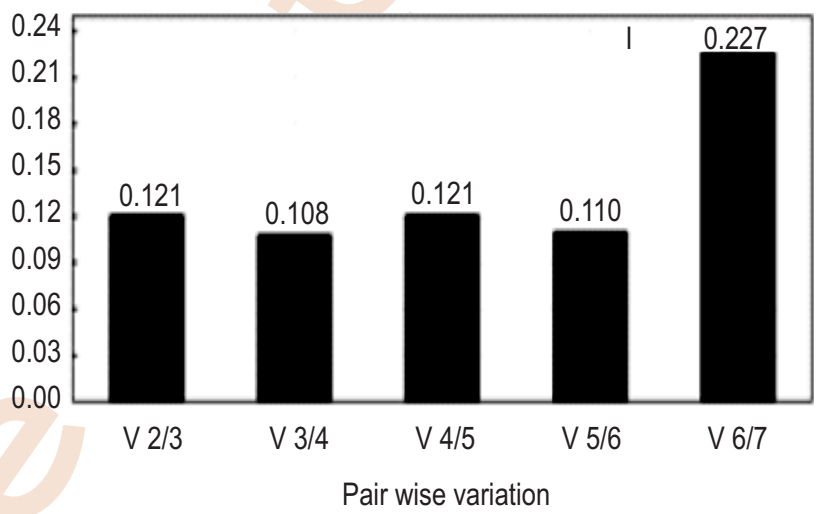

Determination of the optimal number of control genes for normalization

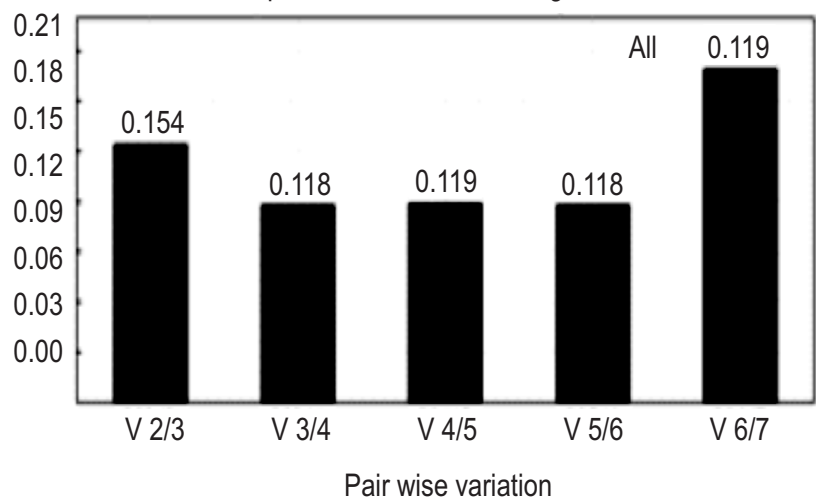

Fig. 2 : Average expression stability values(M) and optimal number of candidate reference genes calculated by geNorm in upright samples (U), inverted samples $(I)$ and total samples $(U+I)$.

additional reference gene is not required (Vandesompele et al., 2002). As shown in Fig. 2, V2/3<0.15 in inverted samples (I), while $\mathrm{V} 2 / 3>0.15$ and $\mathrm{V} 3 / 4<0.15$ in upright samples $(\mathrm{U})$ and in total samples $(U+I)$. Therefore, the upright samples $(U)$ and total samples $(U+I)$ require the top three most stable reference genes (PD-E1/TUA2, HIS) to normalize gene expression, the inverted samples (I) just require the top two most stable reference genes
(PD-E1/TUA2) to normalize gene expression.

Normfinder analysis: Norm Finder is another VBA applet for identifying the optimal normalization gene among a set of candidates. It ranks the set of candidate normalization genes according to their expression stability in a given sample set and given experimental design. Genes with lower stability values 

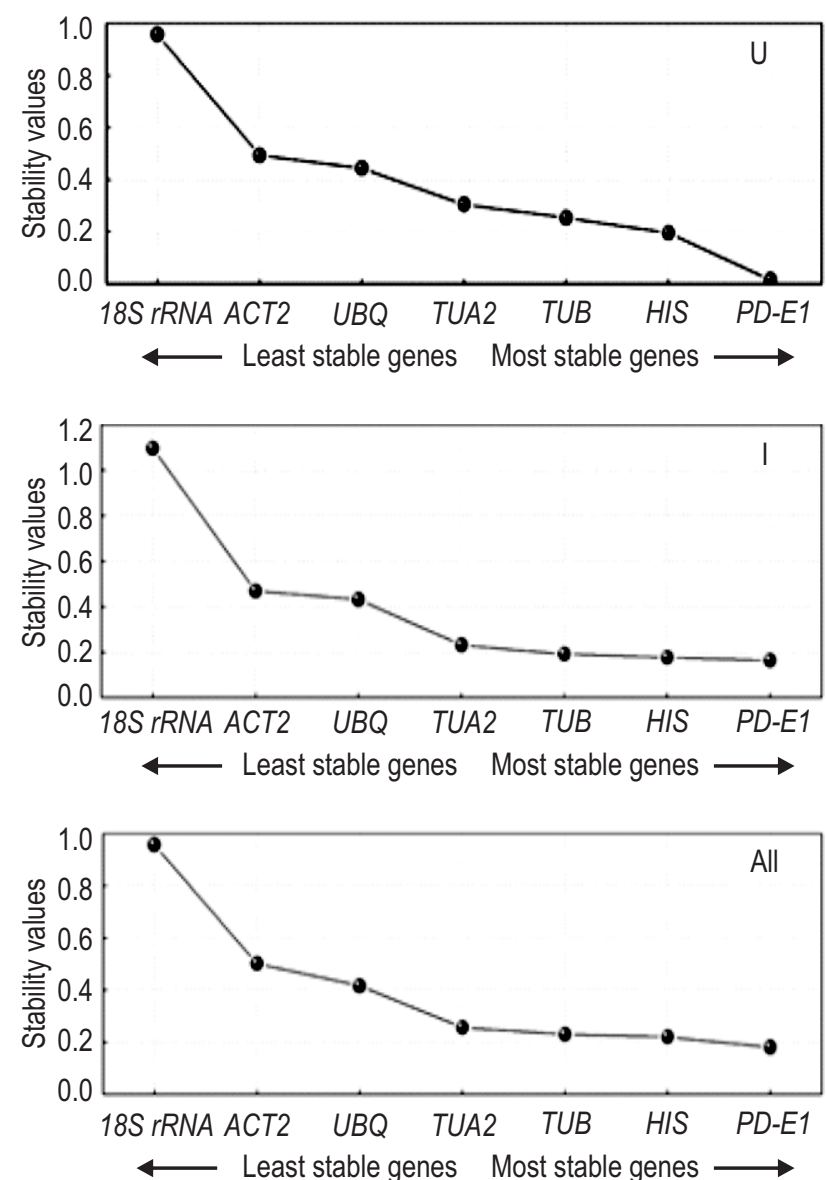

Fig. 3 : Expression stability values of candidate reference genes calculated by NormFinder in upright samples (U), inverted samples (I) and total samples $(U+I)$.

showed less varied expressions and had a stable expressed pattern, while genes with higher stability values showed more varied expressions and had least stable expressed pattern (Andersen et al., 2004). As shown in Fig. 3, the range of genes expression stability was as follows (from the most stable to the least stable): PD-E1, HIS, TUB, TUA2, UBQ, ACT2, 18S rRNA in upright samples $(U)$ and in total samples $(U+I)$, where, $P D-E 1$, TUA2, TUB, HIS, ACT2, UBQ, 18S rRNA in inverted samples (I). However, PD-E1 was the most stable gene in all three conditions.

Best keeper analysis: Best keeper is an Excel-based tool determining the "optimal" HKGs by using a pair-wise correlation analysis of all pairs of candidate genes (less than 10 genes), and calculating the geometric mean of the "best" suited ones. Best keeper index of the coefficient of correlation ( $r$ ), coefficient of variance $(\mathrm{CV})$ and standard deviation (SD) calculated between each gene by comparing raw $\mathrm{Ct}$ values was performed to determine the stable and suitable reference gene. Genes with higher $r$ values had similar overall expression patterns and were more suitable to normalize. The more stable reference gene for accurate normalization of RT-qPCR data was mainly determined by lower CV and SD value. In addition, genes with SD value greater than 1 were unstable (Pfaffl et al., 2004). As shown in Table 2, the SD values of $18 S$ rRNA were greater than 1 under each three condition, indicating that the 18S rRNA was unstable to normalize the transcription of mRNAlevel.

Considering the upright samples (U), HIS and UBQ were the top two most stable genes with low $C V$ value and $S D$ value, followed by $P D-E 1$, as the third one. However, UBQ with the lowest $r$ value had no similar overall expression patterns, which should be avoided. HIS and PD-E1 with higher $r$ value were suitable for reference gene selection. Considering the inverted samples (I), PD-E1 with the lowest CV value, the hightest $r$ value and the second lowest SD value was suitable for reference gene selection. Considering the total samples $(U+I), P D-E 1$ with the lowest $C V$ value, the high test $r$ value and the second lowest $S D$ value was suitable for reference gene selection, similar to inverted samples (I).

Comprehensive analysis: Since different programs showed different ranking results for each gene, Rank Aggreg (Pihur et al., 2009) with non-weighted unsupervised rank aggregation method was used to merge three programs with the aim of obtaining a final list of genes for each condition. The rank positions generated using four statistical approaches were merged, including geNorm $M$ values, Norm finder stability values, Best keeper CV and SD values. The comprehensive results in Fig. 4 indicate that the final optimal lists (from the most stable to the least stable) of the reference genes were HIS, PD-E1, UBQ, TUB, TUA2, ACT2, 18S rRNA in upright samples (U); PD-E1, TUA2, TUB, UBQ, HIS, ACT2, 18S rRNA in inverted samples (I); PD-E1, HIS, TUA2, TUB, UBQ, ACT2, 18S rRNA in total samples $(\mathrm{U}+\mathrm{I})$.

Considering the optimal number of required reference genes calculated by geNorm, combination of multiple reference genes in upright samples $(U)$ should be the top three reference genes, but $U B Q$ with lower r value as the Best keeper showed had no similar overall expression patterns and should be avoided. The final combination of multiple reference genes under diffident conditions were HIS + PD-E1 + TUB in upright samples (U), PD$E 1+T U A 2$ in inverted samples (I), PD-E1 + HIS + TUA2 in total samples $(U+I)$, respectively (Table 3$)$.

RT-qPCR is the most accurate and convenient method for gene expression, which is also used in confirming the results of high-throughput gene differential expression (Peng et al., 2012). Reliable RT-qPCR data depends on suitable reference genes, which must have highly stable expression under different experimental condition (Wang et al., 2015). An increasing number of studies on reference gene selection have been carried out in various plant species (Park et al., 2015; Wang and Lu, 2015; Xu et al., 2015). As for Populus, a model tree for forestry biotechnology, $\mathrm{Xu}$ et al. (2015) considered that EF1a and 18S rRNA were the 
Rang aggregation

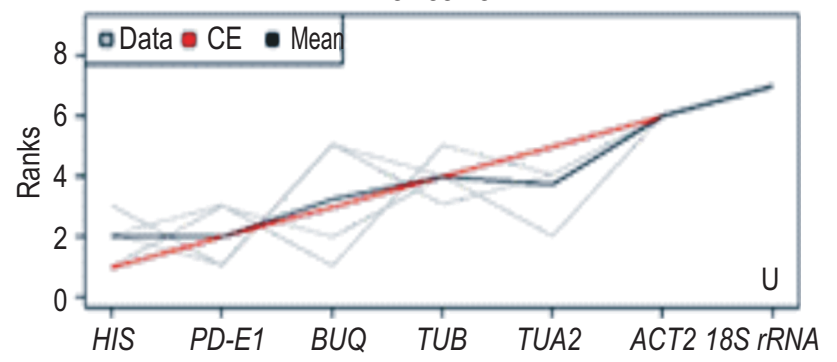

Rang aggregation

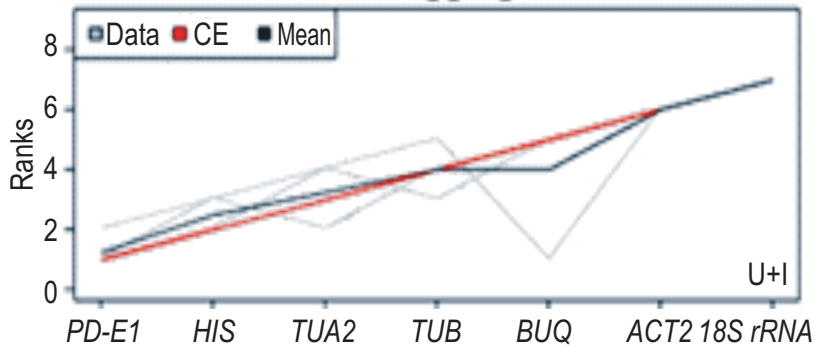

Rang aggregation

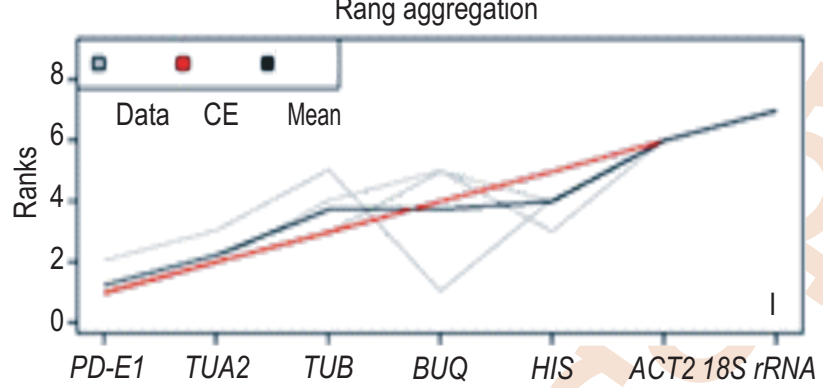

Fig. 4 : Rank aggregation of seven genes lists using the Monte Carlo algorithm in upright samples (U), inverted samples (I) and total samples (U +I). Visual representation of rank aggregation using RankAggreg with the Monte Carlo algorithm and Spearman footrule distances. The seven candidate reference genes were ordered based on their rank position according to four statistical approaches, geNorm M values, NormFinder stability values, BestKeeper CV and SD values (gray lines). The mean rank position of each gene is shown in black, while the model computed using the Monte Carlo algorithm is indicated by a red line.

Table 2: Stability index of candidate reference genes calculated by BestKeeper

\begin{tabular}{|c|c|c|c|c|c|c|c|}
\hline & $P D-E 1$ & HIS & TUA2 & TUB & $U B Q$ & ACT2 & 18S rRNA \\
\hline \multicolumn{8}{|c|}{ Upright samples (U) } \\
\hline r & 0.912 & 0.829 & 0.807 & 0.853 & 0.481 & 0.682 & 0.793 \\
\hline CV & 2.05 & 1.87 & 2.61 & 2.54 & 1.99 & 2.84 & 7.52 \\
\hline SD & 0.54 & 0.42 & 0.63 & 0.66 & 0.41 & 0.72 & 1.21 \\
\hline \multicolumn{8}{|c|}{ Inverted samples (I) } \\
\hline r & 0.913 & 0.877 & 0.914 & 0.890 & 0.668 & 0.821 & 0.853 \\
\hline CV & 2.23 & 2.77 & 2.46 & 2.67 & 2.68 & 3.38 & 10.16 \\
\hline SD & 0.58 & 0.64 & 0.60 & 0.71 & 0.56 & 0.89 & 1.67 \\
\hline \multicolumn{8}{|c|}{ Total samples $(U+I)$} \\
\hline r & 0.875 & 0.841 & 0.834 & 0.867 & 0.637 & 0.746 & 0.777 \\
\hline CV & 1.98 & 2.35 & 2.39 & 2.47 & 2.48 & 3.15 & 7.86 \\
\hline SD & 0.52 & 0.54 & 0.58 & 0.65 & 0.51 & 0.82 & 1.29 \\
\hline
\end{tabular}

r: Coefficient of Correlation; CV: Coefficient of Variance; SD: Standard Deviation

Table 3 : Final combination of multiple reference genes based on geNorm, BestKeeper and RankAggreg

\begin{tabular}{lll}
\hline & \multicolumn{2}{c}{ Three conditions } \\
\hline Upright samples $(U)$ & Inverted samples $(\mathrm{I})$ & Total samples $(\mathrm{U}+\mathrm{I})$ \\
\hline$H I S+P D-E 1+T U B$ & $P D-E 1+T U A 2$ & $P D-E 1+H I S+T U A 2$ \\
\hline
\end{tabular}


appropriate reference genes during Populus root development for RT-qPCR normalization, whereas ACT, HIS and TUB showed relatively low expression stability (Taylor, 2002; Xu et al., 2015). Expression stability of ten candidate reference genes in $P$. euphratica under salt stress revealed different combination of multiple reference genes, Actin $+E F 1 \alpha+G A P D H+R P+U B Q$, $60 S+R P+e I F-5 A+G A P D H, E F 1 \alpha+H I S+R P$ should be used for normalization in roots, stems and leaves, respectively (Wang et al., 2015).

Reference genes were screened in $P$. euphratica leaves under six abiotic stress, the genes selected as optimal for gene expression analysis were RPL17 in ABA, EF1 $\alpha$ in cold, HIS in dehydration, GII in drought and short-duration salt, and TUB in long-duration salt (Wang et al., 2014). Wang et al. (2016) demonstrated that the combination of three most appropriate reference genes, eukaryotic translation initiation factor $5 \mathrm{~A}$ (elF5A), Actin (ACT6) and elongation factor 1-beta (EF1-beta) is suitable for gene expression normalization in $P$. tomentosa stems for both primary and secondary growth. Gutierrez et al. (2008) reported that the expression stability of several genes commonly used were unstable in various tissues of $P$. tremula $\times P$. tremuloides (Gutierrez et al., 2008). However, different Populus species, tissues, experiment treatments lead to no reference genes could be used in common without selection. Meanwhile, it is necessary to select suitable reference genes in $P$. yunnanensis study.

This study is the first report to select and evaluate the stability of several homologous reference genes in barks of $P$. yunnanensis cuttings. At different conditions, different final combination of multiple reference genes were obtained to normalize gene expression in upright samples, inverted samples and total samples, respectively. The results further confirm the change of stability and optimal number of reference genes under different conditions. We recommend using geNorm, Norm Finder, Best keeper, which had the greatest impact and been used widely, to evaluate the stability of candidate reference gene (Tong et al., 2009; Wang et al., 2015). Due to different rank lists obtained by the former three programs, Rank aggreg was performed to weigh the results, which was effective and had been adopted in the latest studies (Pihur et al., 2009; Wang et al., 2014; Wang et al., 2015). In addition, the final combination of multiple reference genes was determined by the optimal number of required reference genes calculated by geNorm and higher $r$ values of the BestKeeper index. In summary, selection of reference genes was a comprehensive result.

The findings of the present study suggest that the combination of HIS + PD-E1 + TUB was suitable for upright samples (U), the combination of $P D-E 1+T U A 2$ was suitable for inverted samples (I), the combination of $P D-E 1+H I S+T U A 2$ was suitable for total samples $(U+I)$ in RT-qPCR analysis. Our results provide an important reference gene selection guide in barks of $P$. yunnanensis cuttings for genes expression research.

\section{Acknowledgments}

The authors would like to thank Xu Gu (Yunnan Academy of Biodiversity, Kunming, China) for providing us Roche LightCycler®96 System. This work was supported by the Natural Science Foundation of China $(31360184,31460205)$.

\section{References}

Abbas, P., Y.Z.H.Y. Hashim and H.M. Salleh: Cytotoxic effects and response surface optimization of solvent extraction of crude extracts from Aquilaria subintegra uninfected branch. Sci. Herit. J., 2,10-15(2018).

Andersen, C.L., J.L. Jensen and T.F. Ørntoft: Normalization of real-time quantitative reverse transcription-PCR data: A model-based variance estimation approach to identify genes suited for normalization, applied to bladder and colon cancer data sets. CancerRes., 64, 5245-5250 (2004).

Brunner, A.M., I.A. Yakovlev and S.H. Strauss: Validating internal controls for quantitative plant gene expression studies. BMC Plant Biol., 4, 14 (2004).

Carraro, N., T.E. Tisdale-Orr, R.M. Clouse, A.S. Knoller and R. Spicer: Diversification and expression of the PIN, AUX/LAX, and ABCB families of putative auxin transporters in Populus. Front Plant Sci., 3, 17 (2012).

Castonguay, Y., J. Michaud and M.P. Dubé: Reference genes for RTqPCR analysis of environmentally and developmentally regulated gene expression in Alfalfa. Am. J. Plant Sci., 6, 132-143 (2015).

Dheda, K., J. Huggett, J. Chang, L. Kim, S. Bustin, M. Johnson, G. Rook and A. Zumla: The implications of using an inappropriate reference gene for real-time reverse transcription PCR data normalization. Anal. Biochem., 344, 141-143 (2005).

Gachon, C., A. Mingam and B. Charrier: Real-time PCR: What relevance to plant studies? J. Exp. Bot., 55, 1445-1454 (2004).

Grbić, M., T. Van Leeuwen, R.M. Clark, S. Rombauts, P. Rouzé, V. Grbić, E.J. Osborne, W. Dermauw, P.C.T. Ngoc and F. Ortego: The genome of Tetranychus urticae reveals herbivorous pest adaptations. Nature, 479, 487-492 (2011).

Grebe, M.: Ups and downs of tissue and planar polarity in plants. BioEssays: News Rev. Mol. Cell. Develop. Biol., 26, 719-729 (2004).

Gutierrez, L., M. Mauriat, S. Guénin, J. Pelloux, J.F. Lefebvre, R. Louvet, C. Rusterucci, T. Moritz, F. Guerineau and C. Bellini: The lack of a systematic validation of reference genes: A serious pitfall undervalued in reverse transcription-polymerase chain reaction (RT-PCR) analysis in plants. Plant Biotechnol. J., 6, 609-618 (2008).

He C.Z., Z.Y. Zhang, B.K. Chen, S.W. Li, A.A. Duan and Y.S. Pu: Preliminary exploration on genetic improvement strategy of Populus yunnanensis. J. West China Forest. Sci., 33, 44-48 (2004).

Khan, A., M. Fiaz, R.A. Khan, J.B. Khan, F.U. Khan and Z. Wahab: Antimicrobial efficacy of Tamarix dioca (L.) leaves and flowers. Sci. Herit. J., 2, 01-03 (2018).

Kibria, A.A., Kamrunnessa and M.M. Rahman: Extraction and evaluation of phytochemicals from green coconut (Cocos nucifera) Shell. Malays. J. Halal Res., 1, 19-22 (2018).

Kumar, V., K. Kumar, R.P. Raman, K.P. Prasad, N. Kumar, S. Kumar, V. Kumar and S. Roy: Evaluation of cellular induction, soluble 
components of proteins and expression of pro-inflammatory genes in Labeo rohita fingerlings. J. Environ. Bio., 39, 486-492 (2018).

Li J.M., T. Yun, J.M. Wang, Y. Zheng, A.P. Zhou, D. Zong and C.Z. He: The polarity of auxin transport in inverted cuttings of Populus yunnanensis. Plant Physiolo., 51, 935-940 (2015).

$\mathrm{Li}, \mathrm{Y}$., P. Yang and $\mathrm{H}$. Wang: Collecting coal fired power environmental tax to promote wind power development and environmental improvement. Acta Sci. Malays., 2, 05-08 (2018).

Lu, Z., X. Daxing and W. Hailun. Two-stage Cubature Kalman Filter and its application in water pollution model. Acta Sci. Malays., 2, 09-13 (2018).

Nolan, T., R.E. Hands and S.A. Bustin: Quantification of mRNA using real-time RT-PCR. Nat. Protoc., 1, 1559-1582 (2006).

Park, S.J., S.G. Kwon, J.H. Hwang, D.H. Park, T.W. Kim and C.W. Kim: Selection of appropriate reference genes for RT-qPCR analysis in Berkshire, Duroc, Landrace and Yorkshire pigs. Gene, 558, 152$158(2015)$

Patil, A.S., R. Thankappan, R. Mehta, R. Yadav, A. Kumar, G.P. Mishra, J.R. Dobaria, P.P. Thirumalaisamy and R.K. Jain: Evaluation of transgenic peanut plants encoding coat protein and nucleocapsid protein genes for resistance to tobacco streak virus and peanut bud necrosis virus. J. Environ. Biol., 38, 187-196 (2017).

Peng, S., H. Jiang, S. Zhang, L. Chen, X. Li, H. Korpelainen and C. Li: Transcriptional profiling reveals sexual differences of the leaf transcriptomes in response to drought stress in Populus yunnanensis. Tree Physiology, 32, 1541-1555 (2012).

Pfaffl, M.W., A. Tichopad, C. Prgomet and T.P. Neuvians: Determination of stable housekeeping genes, differentially regulated target genes and sample integrity: BestKeeper-Excel-based tool using pair-wise correlations. Biotechnol. Lett., 26, 509-515 (2004).

Pihur, V., S. Datta and S. Datta: Rank aggreg, an R package for weighted rank aggregation. BMC Bioinformatics, 10,62 (2009).

Sachs, T.: Cell polarity and tissue patterning in plants. Development, 113 83-93 (1991).

Schmid, H., C.D. Cohen, A. Henger, S. Irrgang, D. Schlöndorff and M. Kretzler: Validation of endogenous controls for gene expression analysis in micro dissected human renal biopsies. Kidney Int., 64 356-360 (2003).

Secchi, F., B. Maclver, M.L. Zeidel and M.A. Zwieniecki: Functional analysis of putative genes encoding the PIP2 water channel subfamily in Populus trichocarpa. Tree Physiology, 29, 1467-77 (2009).
Shuai, P., D. Liang, S. Tang, Z. Zhang, C.Y. Ye, Y. Su, X. Xia and W. Yin: Genome-wide identification and functional prediction of novel and drought-responsive lincRNAs in Populus trichocarpa. J. Exp. Bot., 65, 4975-83 (2014).

Solanas, M., R. Moral and E. Escrich: Unsuitability of using ribosomal RNA as loading control for Northern blot analyses related to the imbalance between messenger and ribosomal RNA content in rat mammary tumors. Anal. Biochem., 288, 99-102 (2001).

Sultana, T., S. Rahman, N. Naher, R.M. Masum, A.H.A. Ahmed and R. Islam: Performance of fruit vegetables in summer under mahagony based agroforestry systems. Malays. J. Halal Res., 1, 08-14 (2018).

Suzuki, T., P.J. Higgins and D.R. Crawford: Control selection for RNA quantitation. Biotechniques, 29, 332-7 (2000).

Taylor, G: Populus: Arabidopsis for forestry. Do we need a model tree? Ann. Bot., 90, 681-689 (2002)

Tong, Z., Z. Gao, F. Wang, J. Zhou and Z. Zhang: Selection of reliable reference genes for gene expression studies in peach using realtime PCR. BMC Mol. Biol., 10, 71 (2009).

Vandesompele, J., K. De Preter, F. Pattyn, B. Poppe, N. Van Roy, A. De Paepe and F. Speleman: Accurate normalization of real-time quantitative RT-PCR data by geometric averaging of multiple internal control genes. Genome Biol., 3, 0034 (2002).

Wang, H.L., J. Chen, Q. Tian, S. Wang, X. Xia and W. Yin: Identification and validation of reference genes for Populus euphratica gene expression analysis during abiotic stresses by quantitative realtime PCR. Physiol. Plant., 152, 529-45 (2014).

Wang, H.L., L. Li, S. Tang, C. Yuan, Q. Tian, Y. Su, H.G. Li, L. Zhao, W. Yin, R. Zhao and X. Xia: Evaluation of appropriate reference genes for reverse transcription-quantitative PCR studies in different tissues of a desert poplar via comparision of different algorithms. Int. J. Mol. Sci., 16, 20468-20491 (2015).

Wang, M. and S. Lu:: Validation of suitable reference genes for quantitative gene expression analysis in Panax ginseng. Front. Plant Sci., 6, 1259 (2015).

Wang, Y., Y. Chen, L. Ding, J. Zhang, J. Wei and H. Wang: Validation of reference genes for gene expression by quantitative real-time RTPCR in stem segments spanning primary to secondary growth in Populustomentosa. PloS One, 11, e0157370 (2016).

Xu, Y., H. Li, X. Li, J. Lin, Z. Wang, Q. Yang and Y. Chang: Systematic selection and validation of appropriate reference genes for gene expression studies by quantitative real-time PCR in pear. Acta Physiol. Plant., 37, 40 (2015). 